\title{
Asociación entre el triángulo hioideo negativo y la respiración oral
}

\section{Association between the negative hyoid triangle and oral breathing}

\section{Resumen}

Objetivo. Evaluar la asociación entre la presencia del triángulo hioideo negativo y la respiración oral. Métodos. Se analizó un total de 100 telerradiografías de perfil, 50 de pacientes con respiración nasal y 50 con respiración oral, $70 \%$ de pacientes de sexo femenino y $30 \%$ masculino. El 59\% correspondió a pacientes de entre 20 a 30 años de edad, el 21\% de entre 10-20 ańos, el 15\% de entre 30-40 años y el 5\% de entre 40-50 ańos. Los criterios de inclusión fueron fichas clínicas de pacientes que posean telerradiografías de perfil con registro del tipo de respiración (oral o nasal). Los criterios de exclusión fueron fichas clínicas de pacientes con telerradiografía en formato digital, con errores de posicionamiento o dańadas o de sujetos con síndromes genéticos, sin tratamiento de ortodoncia/ ortopedia previos. Se realizó trazado cefalométrico manual (técnica de Rocabado). Se aplicó análisis estadístico descriptivo y prueba estadística $\mathrm{Chi}^{2}$ de Pearson a través de tablas de contingencia. Resultados. Se detectó que existe una asociación estadísticamente significativa $(\mathrm{p}<0,001)$ entre la presencia de un triángulo hioideo negativo y la respiración oral, siendo los pacientes de sexo femenino $(\mathrm{p}<0,001)$ y de 10 a 20 años de edad los que la presentaron con mayor frecuencia $(81 \%)$. Conclusiones. En la población de estudio analizada existe una asociación estadísticamente significativa entre la presencia de triángulo hioideo negativo y la respiración oral, lo que parece estar relacionado con el sexo y la edad.

Palabras clave: Respiración por la boca; Hueso hioides; Radiografía (fuente: DeCS BIREME).

\begin{abstract}
Objective. The objective of this study was to evaluate the association between the presence of the hyoid triangle negative and oral breathing. Methods. We analyzed a total of 100 lateral teleradiographs, 50 belonging to patients with oral breathing and 50 with nasal breathing. Of these, $70 \%$ corresponded to female patients $(n=70)$ and $30 \%$ to male patients (30). 59\% corresponded to patients between 20 and 30 years, $21 \%$ between $10-20$ years, $15 \%$ between $30-40$ years and $5 \%$ between $40-50$ years old. The inclusion criteria were clinical records of patients of both sexes between 15 and 50 years of age,
\end{abstract}

\section{Artículo Original}

Daniela Flores Toro 1,a, Joel Bravo Bown 1,a,b

1 Universidad de Antofagasta, Facultad de Medicina y Odontología, Departamento de Odontología, Antofagasta, Chile.

a Cirujano Dentista.

${ }^{\mathrm{b}}$ Magíster en Ciencias Odontológicas.

\section{Correspondencia:}

Joel Bravo Bown: joel.bravo@uantof.cl

Avenida Universidad de Antofagasta 02800, Comuna de

Antofagasta, Antofagasta- Chile

ORCID: 0000-0001-8816-0161

\section{Coautores:}

Daniela Flores Toro: dani.flores.toro@gmail.com

\section{Editora:}

Rosse Mary Falcón-Antenucci

Universidad Inca Garcilaso de la Vega, Lima, Perú.

Conflicto de intereses: los autores declaran no tener conflictos de interés.

Fuente de financiamiento: autofinanciado

Recibido: 25/11/19

Aceptado: $16 / 02 / 20$

Publicado: 09/05/20

(C) Los autores. Este artículo es publicado por la revista Odontología Sanmarquina de la Facultad de Odontología, Universidad Nacional Mayor de San Marcos. Este es un artículo de acceso abierto, distribuido bajo los términos de la licencia Creative Commons Atribucion - No Comercia_Compartir Igual 4.0 Internacional. (http://creativecommons.org/licenses/by-nc-sa/4.0/) que permite el uso no comercial, distribución y reproducción en cualquier medio, siempre que la obra original sea debidamente citada. 
who have profile teleradiographs and in which their type of breathing (oral or nasal) has been recorded. The exclusion criteria were clinical records of patients in whom the teleradiography is in digital format, with positioning or damaged errors or belonging to subjects who have diagnosed genetic syndromes and who have not received previous orthodontic/orthopedic treatment. The $\mathrm{x}$-rays were analyzed by cephalometric technique of Rocabado. Descriptive statistical analysis was performed and $\mathrm{Chi}^{2}$ statistical test through contingency tables. Results. It was found that there is a statistically significant association $(\mathrm{p}<0.001)$ between the presence of a triangle hyoid negative and oral breathing, being the female patients $(\mathrm{p}<0.001)$ of 10 to 20 years which were more frequent $(81 \%)$. Conclusions. It was possible to conclude that there is a statistically significant association between the presence of negative hyoid triangle and oral breathing, which seems to be related to sex and age.

Keywords: Mouth breathing; Hyoid bone; Radiography (source: MeSH NLM).

\section{Introducción}

El hioides es un hueso impar, situado en la línea media, cóncavo posteriormente y con forma de herradura, se ubica en el cuello, suspendido por músculos y ligamentos, entre el suelo de la boca y la laringe. Forma parte del complejo hio-gloso-faríngeo, prestando inserción a estructuras provenientes de faringe, lengua, mandíbula y cráneo ${ }^{1}$. Es un hueso móvil que responde a los requerimientos funcionales durante la masticación, deglución, respiración, fonación ${ }^{2}$ y debe permanecer en equilibrio entre las tensiones musculares, proporcionando un punto de apoyo para la acción de los distintos músculos. Participa en el mantenimiento de la vía aérea, al producir la tensión de la fascia cervical, disminuyendo así la succión interna de las partes blandas e impidiendo la compresión de grandes vasos y los pulmones, por todo lo anterior, es que una mala posición del hueso hioides podría producir un desequilibrio en cualquiera de las funciones antes mencionadas ${ }^{3,4}$. Las medidas cefalométricas para el análisis de la posición hioidea incluyen medidas respecto a planos de referencia craneales y respecto al plano mandibular (PM), el análisis más frecuentemente usado es el de Rocabado ${ }^{5}$. Este análisis se realiza uniendo los puntos cefalométricos $\mathrm{H}$ (hyoidale, punto más anterosuperior del cuerpo del hioides), RGn (retrognation, punto más posteroinferior de la sínfisis mandibular) y C3 (punto más anteroinferior del cuerpo de C3). En base a esto, se puede calcular: $\mathrm{H}-\mathrm{H}^{\prime}$ : que es la distancia lineal entre los puntos $\mathrm{H}$ y H'. H' es la proyección perpendicular de $\mathrm{H}$ sobre el plano C3-RGn, esta medida representa la posición vertical del hioides respecto a la columna cervical y la sínfisis mandibular. H-RGn: distancia lineal entre H y RGn, representa la posición anteroposterior del hioides respecto a la sínfisis mandibular. H-C3: Distancia lineal entre $\mathrm{H}$ y C3, representa la posición anteroposterior del hioides respecto a la columna cervical. En una situación normal, el hioides debería estar situado verticalmente por debajo del plano C3-RGn (hasta $5 \mathrm{~mm}$ ), lo que daría como resultado un triángulo "positivo". Si alguna alteración provoca la elevación del hioides, se encontrará sobre el plano C3-RGn (provocando la desaparición del triángulo) o por encima de él (formándose un triángulo "negativo").
La asociación entre la posición negativa del triángulo hioideo y la respiración oral es de suma importancia en el diagnóstico funcional de los pacientes, ya que una postura errónea del hueso hioides produce además cambios en la posición de la lengua, disminuyendo la vía aérea superior, lo que a su vez influye en la posición dentaria. Esta asociación es de vital importancia en las derivaciones para el tratamiento de la respiración oral a otras áreas de la salud, como fonoaudiología, otorrinolaringología y kinesiología 6 .

La respiración oral o bucal es una alteración común en la infancia y tiene una etiología multifactorial. Normalmente, los niños afectados no tienen una respiración oral exclusiva sino un patrón mixto, en el que predomina la ventilación a través de la cavidad oral en lugar de la vía normal nasal ${ }^{7}$. Existe evidencia limitada y controversial con respecto a la posición del triángulo hioideo y su asociación con la respiración oral. Algunos autores ${ }^{6,7}$ no han encontrado diferencias en la posición del hioides en los individuos con respiración oral, concluyendo que el hioides tiene una posición estable y no se afecta por las alteraciones posturales debidas a la respiración, perdiendo su posible valor diagnóstico. Ferraz ${ }^{6}$ y Cuccia ${ }^{7}$ no encontraron una asociación estadísticamente significativa entre la posición hioidea y el patrón respiratorio, y concluyeron que el hioides se mantiene en una posición estable a nivel del plano que une el punto más anteroinferior del cuerpo de C3 y el punto más posteroinferior de la sínfisis mandibular (C3-RGn). Mencionaron además que existen pacientes que para asegurar las correctas proporciones del espacio aéreo sufren una adaptación postural orofaríngea para mantener la vía aérea, sin embargo, otros autores, han encontrado que la posición del hioides sí varía en función del tipo respiratorio, señalando que los niños con asma y respiración oral presentan con mayor frecuencia un triángulo hioideo ausente o negativo ${ }^{8-10}$.

El propósito de este trabajo de investigación fue evaluar la asociación entre la posición del hueso hioideo y la respiración oral, con el fin de determinar si su posición se encuentra alterada en la respiración oral.

\section{Métodos}

Diseño del estudio. Se realizó un estudio de naturaleza observacional, transversal y analítico, que comenzó en 
marzo 2018 y terminó en diciembre 2018. Todos los sujetos menores de 18 años debieron firmar un asentimiento informado y todos los mayores de 18 ańos un consentimiento informado. Ambos documentos fueron aprobados por el Comité de ética en investigación Científica de la Universidad de Antofagasta (Folio $\mathrm{N}^{\circ}$ 153/2018).

Universo. El Universo estuvo conformado por 100 telerradiografías de perfil, 50 de ellas pertenecientes a pacientes con respiración nasal y 50 con respiración oral (grupo control). Las fichas clínicas fueron reclutadas desde la clínica de Postítulo de Ortodoncia y Ortopedia Dentomaxilar en la Universidad de Antofagasta (Antofagasta, Chile).

Criterios de inclusión y exclusión. Los criterios de inclusión fueron: fichas clínicas de pacientes de ambos sexos de entre 15 a 50 ańos de edad, que posean telerradiografías de perfil y en las que se haya registrado su tipo de respiración (oral o nasal). Los criterios de exclusión fueron: fichas clínicas de pacientes en los que la telerradiografía se encuentre en formato digital, con errores de posicionamiento o dańadas o que pertenezcan a sujetos que presenten síndromes genéticos diagnosticados y que no hayan recibido tratamiento de ortodoncia/ortopedia previos.

Calibración y registro de variables. Se realizó una calibración intra-examinador analizando un total de 50 telerradiografías de perfil, obteniendo un nivel de concordancia de 0,92 (índice kappa de Cohen) en la comparación de los trazados cefalométricos. El registro de las variables se realizó en una ficha de registro ad hoc se registró la variable sexo, edad, y se procedió a realizar un trazado cefalométrico manual, utilizando la técnica de Rocabado ${ }^{5}$. Cada trazado manual se realizó sobre film transparente Photocop Fil de Staedler (Staedtler Mars GmbH \& Co. KG, Alemania, Nuremberg) utilizando rotuladores permanentes Staedler de 0,5 color negro para el trazado, y color azul para las medidas.

Análisis estadístico. Se realizó análisis estadístico descriptivo, en las variables categóricas se aplicó la prueba estadística $\mathrm{Chi}^{2}$ de Pearson a través de tablas de contingencia. Se utilizó software SPSS v.22. (SPSS Inc., IBM, Chicago, IL, USA).

\section{Resultados}

Características sociodemográficas. En la Tabla 1 se presentan las variables sexo y edad. De un total de 100 fichas clínicas analizadas, 70 correspondieron al sexo femenino (70\%) y 30 al sexo masculino (30\%). La edad se agrupó en intervalos, siendo la más frecuente aquella que va entre los 20 a 30 ańos (59\%).

Comparación de la presencia de triángulo hioideo negativo y positivo en los grupos de respiración oral y nasal. En la Tabla 2 se presenta la comparación entre los grupos según respiración y el triángulo hioideo. Se detectó que existe una asociación estadísticamente significativa $(\mathrm{p}<0,001)$ entre la presencia de un triángulo hioideo negativo y la respiración oral.

Comparación de la presencia de triángulo hioideo negativo y positivo en distintos grupos etarios. En la Tabla 3 se presenta la comparación de la presencia de triángulo hioideo negativo y positivo en distintos grupos etarios según rangos de edad. Se detectó que existen diferencias estadísticamente significativas entre los distintos

Tabla 1. Características sociodemográficas (sexo y rango de edad). Para cada variable se presentan sus frecuencias absolutas $(\mathrm{n})$ y relativas $(\%)$

\begin{tabular}{lcc}
\hline Sexo & $\mathbf{n}$ & $\%$ \\
\hline Femenino & 70 & 70,0 \\
Masculino & 30 & 30,0 \\
Rango de edad (años) & & 21,0 \\
\hline $10-20$ & 21 & 59,0 \\
$20-30$ & 59 & 15,0 \\
$30-40$ & 15 & 5,0 \\
$40-50$ & 5 & 5 \\
\hline
\end{tabular}

Tabla 2. Comparación de los trazados realizados que determinaron la presencia de triángulo hioideo negativo y positivo en los grupos de respiración oral y nasal. Para cada variable se presentan sus frecuencias absolutas ( $n$ ) y relativas (\%)

\begin{tabular}{|c|c|c|c|c|c|c|}
\hline \multirow[b]{3}{*}{ Triángulo hioideo } & \multicolumn{4}{|c|}{ Respiración } & & \\
\hline & \multicolumn{2}{|c|}{ oral } & \multicolumn{2}{|c|}{ nasal } & \multicolumn{2}{|c|}{ Total } \\
\hline & $\mathbf{n}$ & $\%$ & $\mathbf{n}$ & $\%$ & n & $\%$ \\
\hline Negativo & 42 & 42,0 & 12 & 12,0 & 46 & 46,0 \\
\hline Positivo & 8 & 8,0 & 38 & 38,0 & 54 & 54,0 \\
\hline Total & 50 & 50,0 & 50 & 50,0 & 100 & 100,0 \\
\hline
\end{tabular}

Significancia estadística; basado en la prueba $\mathrm{Chi}^{2}(\mathrm{p}<0,001)$ 
grupos etarios $(\mathrm{p}=0,01)$ siendo los pacientes de entre 10 20 años de edad los que presentan más frecuentemente un triángulo hioideo negativo (81\%).

Comparación de la presencia de triángulo hioideo negativo y positivo según sexo. En la Tabla 4 se presenta la comparación de la presencia de triángulo hioideo negativo y positivo en la variable sexo. Se pudo detectar la existencia de una asociación estadísticamente significativa entre la presencia de un triángulo hioideo negativo y el sexo femenino $(p<0,001)$.

\section{Discusión}

El estudio de la posición hioidea permite la evaluación de su posición fisiológica y las funciones de la anatomía que lo rodea, pudiendo ser útil en la confirmación del mal hábito de respiración oral. Siempre que se realiza un tratamiento de ortodoncia, además de corregir la maloclusión, se debería corregir también el mal hábito, generando un cambio en la posición del hueso hioides, de manera que adopte una nueva posición estable, permitiendo al especialista descartar la posibilidad de recidiva.

Los resultados del presente estudio concuerdan con $\mathrm{Mu}$ ńoz et al. ${ }^{10}$, quien reportó una asociación positiva entre la posición del hueso hioideo y la respiración oral. La mayoría de los respiradores nasales tuvieron un triángulo hioideo positivo, mientras que la mayoría de los respiradores orales tuvieron un triángulo hioideo negativo, con el punto $\mathrm{H}$ por encima o sobre el plano C3-RGn (hioides en una posición más elevada). Esto es concordante con Chaves et al. ${ }^{9}$ quien reportó que los niños con asma y respiración oral presentan con mayor frecuencia un triángulo hioideo negativo.
Ferraz $^{6}$ y Cuccia $^{7}$ no encontraron una asociación estadísticamente significativa entre la posición hioidea y el patrón respiratorio, y concluyeron que el hioides se mantiene en una posición estable a nivel de C3-RGn. Mencionaron además que existen pacientes que para asegurar las correctas proporciones del espacio aéreo sufren una adaptación postural orofaríngea para mantener la vía aérea.

La más alta posición del hioides puede deberse a cambios musculares en el área circundante o por cambios en los puntos de referencia cervical o mandibular, ya que estas estructuras se encuentran alteradas en su posición en los respiradores orales (al descender la mandíbula el hioides estaría elevado respecto a ella). Esta asociación se debería a que la posición del hioides es reflejo de las tensiones de los músculos, ligamentos y fascias unidos a él, la unión funcional de este hueso con el cráneo, la mandíbula y la lengua.

Según Tourné et al. ${ }^{12}$, en los pacientes con respiración oral existe una obstrucción de la vía aérea, lo que provoca un descenso de la lengua y una serie de adaptaciones posturales a nivel orofaríngeo. Además, se produce una postero-rotación mandibular, sin embargo, el hioides no sigue el mismo movimiento que la sínfisis, porque si sucediera eso, invadiría el espacio vital orofaríngeo, por lo que la estabilidad del espacio de la vía aérea es el principal factor determinante de la posición del hioides.

En el presente estudio se encontró una mayor asociación entre la presencia de un triángulo hioideo negativo en mujeres con respiración oral, esto puede deberse a que la postura se produce como un mecanismo de adaptación y se mantiene como respuesta a una condición

Tabla 3. Comparación de los trazados realizados que determinaron la presencia de triángulo hioideo negativo y positivo en los distintos grupos etáreos (rangos). Para cada variable se presentan sus frecuencias absolutas (n) y relativas (\%)

\begin{tabular}{|c|c|c|c|c|c|c|c|c|c|c|}
\hline \multirow[b]{3}{*}{ Triángulo hioideo } & \multicolumn{8}{|c|}{ Edad (años) } & & \\
\hline & \multicolumn{2}{|c|}{$10-20$} & \multicolumn{2}{|c|}{$20-30$} & \multicolumn{2}{|c|}{$30-40$} & \multicolumn{2}{|c|}{$40-50$} & \multicolumn{2}{|c|}{ Total } \\
\hline & $\mathrm{n}$ & $\%$ & $\mathrm{n}$ & $\%$ & $\mathrm{n}$ & $\%$ & $\mathrm{n}$ & $\%$ & n & $\%$ \\
\hline negativo & 17 & 81,0 & 25 & 42,4 & 9 & 60,0 & 3 & 60,0 & 54 & 54,0 \\
\hline positivo & 4 & 19,0 & 34 & 57,6 & 6 & 40,0 & 2 & 40,0 & 46 & 46,0 \\
\hline Total & 21 & 100,0 & 59 & 100,0 & 15 & 100,0 & 5 & 100,0 & 100 & 100,0 \\
\hline
\end{tabular}

Significancia estadística; basado en la prueba Chi2 $(p=0,017)$

Tabla 4. Comparación de la presencia de triángulo hioideo negativo y positivo según sexo. Se presentan para cada variable sus frecuencias absolutas $(\mathrm{n})$ y relativas $(\%)$

\begin{tabular}{|c|c|c|c|c|c|c|}
\hline \multirow[b]{3}{*}{ Triángulo hioideo } & \multicolumn{6}{|c|}{ Sexo } \\
\hline & \multicolumn{2}{|c|}{ femenino } & \multicolumn{2}{|c|}{ masculino } & \multicolumn{2}{|c|}{ Total } \\
\hline & $\mathbf{n}$ & $\%$ & $\mathbf{n}$ & $\%$ & $\mathbf{n}$ & $\%$ \\
\hline negativo & 45 & 64,3 & 5 & 16,7 & 50 & 50,0 \\
\hline positivo & 25 & 35,7 & 25 & 83,3 & 50 & 50,0 \\
\hline Total & 70 & 100,0 & 30 & 100,0 & 100 & 100,0 \\
\hline
\end{tabular}

Significancia estadística; basado en la prueba $\mathrm{Chi}^{2}(\mathrm{p}<0,001)$ 
patológica o psico-emocional, la que habitualmente es más prevalente en mujeres debido a su nivel y carga emocional más activa, lo que influiría en el crecimiento craneofacial y determinaría patrones morfoesqueletales y neuromusculares que generan una tensión exagerada de la musculatura supra e infrahioídea, lo que a su vez ocasionaría tensión a nivel del hueso hioides produciendo un desequilibrio muscular ${ }^{13}$.

De acuerdo con la muestra analizada es posible concluir que existe una asociación estadísticamente significativa entre la presencia de triángulo hioideo negativo y la respiración oral, lo que parece estar relacionado con el sexo y la edad.

\section{Referencias bibliográficas}

1. Velayos JL. Estructuras óseas y cartilaginosas. Fosas craneofaciales. En: Velayos JL, editor. Anatomía de la Cabeza para odontólogos. $4 \mathrm{a}$ ed. Madrid: Editorial Médica Panamericana; 2007. p. 31-88.

2. Schünke M, Schulte E, Schumacher U. Cavidad oral y regiones periorales. En: Baker EW, editor. Prometheus Atlas de Anatomía de la cabeza y el cuello. New York: Thieme Medical Publishers; 2010. p. 178-222.

3. Norton NS. Cuello. En: Norton NS, editor. Netter Anatomía de cabeza y cuello para odontólogos. Barcelona: Elsevier Masson; 2007. p. 111-58.

4. Henríquez J, Sandoval P, Fuentes R. Radiological anatomy of the hyoid bone. Rev Chil anat. 2000;18(1):117-24.

5. Rocabado MS. Relaciones biomecânicas de las regiones craneales, cervicales e hióideas. Ortodoncia, 1994;58(115):51-6.
6. Ferraz M, Sousa M, Pereira J, Nouer D, Magnani M. Avaliação cefalométrica o triângulo hióideo em brasileiros da região de Piracicaba. In: Anais da Reuniáo da Sociedade Brasileira de Pesquisa Odontológica, 2003. Águas de Lindóia. São Paulo: SBPqO; 2003. p.161.

7. Cuccia A, Lotti M, Caradonna D. Oral breathing and head posture. Angle Orthod. 2008;78(1):77-82.

8. Bibby, R. The hyoid bone position in mouth breathers and tongue-thrusters. Am J Orthod. 1984;85(5):431-3.

9. Chaves T, de Andrade T, Monteiro S, Watanabe P, Oliveira A, Grossi D. Craniocervical posture and hyoid bone position in children with mild and moderate asthma and mouth breathing. Int J Pediatr Otorhinolaryngol. 2010;74(9):1021-7.

10. Muñoz ICL, Orta PB. Comparison of cephalometric patterns in mouth breathing and nose breathing children. Int J Pediatr Otorhinolaryngol. 2014;78(7):116772 .

11. Bolzan G de P, Souza JA, Boton L de M, Silva AMT da, Corrêa ECR. Facial type and head posture of nasal and mouth-breathing children. J Soc Bras Fonoaudiol. 2011;23(4):315-20.

12. Tourne L, Schweiger J. Immediate postural responses to total nasal obstruction. Am J Orthod Dentofacial Orthop. 1996;110(6):606-11.

13. Garcia F, Mussolino A. Alteraciones posturales y su repercusión en el sistema estomatognático. Acta Odont Venez. 2008;46(4):1-7. 
